\title{
Heralded quantum entanglement between two crystals
}

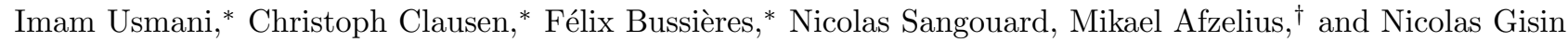 \\ Group of Applied Physics, University of Geneva, CH-1211 Geneva 4, Switzerland
}

(Dated: 24 August 2011)

\begin{abstract}
Quantum networks require the crucial ability to entangle quantum nodes [1. A prominent example is the quantum repeater 2 4 , which allows overcoming the distance barrier of direct transmission of single photons, provided remote quantum memories can be entangled in a heralded fashion. Here we report the observation of heralded entanglement between two ensembles of rare-earth-ions doped into separate crystals. A heralded single photon is sent through a 50/50 beamsplitter, creating a single-photon entangled state delocalized between two spatial modes. The quantum state of each mode is subsequently mapped onto a crystal, leading to an entangled state consisting of a single collective excitation delocalized between two crystals. This entanglement is revealed by mapping it back to optical modes and by estimating the concurrence of the retrieved light state [5]. Our results highlight the potential of rare-earth-ions doped crystals for entangled quantum nodes and bring quantum networks based on solid-state resources one step closer.
\end{abstract}

Quantum entanglement challenges our intuition about physical reality. At the same time, it is an essential ingredient in quantum communication [6], quantum precision measurements [7] and quantum computing [8, 9]. In quantum communication, photons are naturally used as carriers of entanglement using either free-space or optical fiber transmission. Yet, even with ultra low-loss telecommunication optical fiber, the transmission probability decreases exponentially with distance, limiting the achievable communication distance to a few hundred kilometers [10]. A potential solution is to use quantum repeaters 2 2 based on linear optics and quantum memories [3, 4] with which the entanglement distribution time scales polynomially with the transmission distance, provided entanglement between quantum memories in remote locations can be heralded. In this context, one can more generally consider prospective quantum networks [1] where nodes generate, process and store quantum information, while photons transport quantum states from site to site and distribute entanglement over the entire network. An essential step towards the implementation of such potential technologies is to create entanglement between two quantum memories in an heralded manner [3, 4].

Experimental observation of heralded entanglement between two independent atomic systems for quantum networks was achieved using cold gas ensembles involving either two distinct ensembles [5], or two spatial modes in the same ensemble [11 15] (note that in ref. 15] the entanglement was postselected). Complete elementary links of a quantum repeater (based on heralded entanglement) have been implemented in cold gas systems [16, 17. and with two trapped ions [18. For the realization of scalable quantum repeaters, solid-state devices are technologically appealing [19. In this context, important results have already been obtained with rare-earth-ion (RE) doped crystals used as quantum memories. These

\footnotetext{
* These authors contributed equally to this work.

$\dagger$ mikael.afzelius@unige.ch
}

results include light storage times greater than one second [20], storage efficiency of $69 \%$ [21] and quantum storage of 64 independent optical modes in one crystal 22 . (see also ref. 23]). Recent achievements [24, 25] include the storage, in a single RE-doped crystal, of photonic time-bin entanglement generated through spontaneous parametric downconversion (SPDC). Heralded entanglement between two RE-doped crystals using an SPDC source, a common resource in quantum optics, represents an important step towards the implementation of quantum repeater architectures based on solid-state devices [26].

Here we present the observation of heralded quantum entanglement between two neodymium ensembles doped in two yttrium ortho-silicate crystals $\left(\mathrm{Nd}^{3+}: \mathrm{Y}_{2} \mathrm{SiO}_{5}\right)$ separated by 1.3 centimeters. Let us conceptually detail our experiment, depicted in Fig. 1. A nonlinear optical waveguide is pumped to produce photon pairs through SPDC. The resulting idler $(1338 \mathrm{~nm})$ and signal $(883 \mathrm{~nm})$ photons are strongly filtered to match the absorption bandwidth of the crystals, yielding a coherence time of $7 \mathrm{~ns}$. In the limit where the probability of creating a single pair is much smaller than one, the detection of an idler photon heralds the presence of a single signal photon. By sending the latter through a balanced beamsplitter, one ideally heralds a single-photon entangled state 28] $\frac{1}{\sqrt{2}}\left(|1\rangle_{A}|0\rangle_{B}+|0\rangle_{A}|1\rangle_{B}\right)$ between the two spatial output modes $A$ and $B$. In each of the modes a crystal acts as quantum memory, denoted $M_{A}$ or $M_{B}$. Upon absorption of the single photon by one of the crystals [29], the detection of an idler photon heralds the creation of a single collective excitation delocalized between the two crystals, ideally written as $\frac{1}{\sqrt{2}}\left(|W\rangle_{A}|0\rangle_{B}+|0\rangle_{A}|W\rangle_{B}\right)$. Here, $|W\rangle_{A}$ (or $|W\rangle_{B}$ ) is the Dicke-like state created by the absorption of a single photon in $M_{A}$ (or $M_{B}$ ). To determine the presence of entanglement between the memories, we use a photon echo technique based on an atomic frequency comb 29 33. that reconverts the collective excitation into optical modes $A$ and $B$ after a preprogrammed storage time of $33 \mathrm{~ns}$. Note that the memories are used in a double-pass configuration, which effectively increases the 


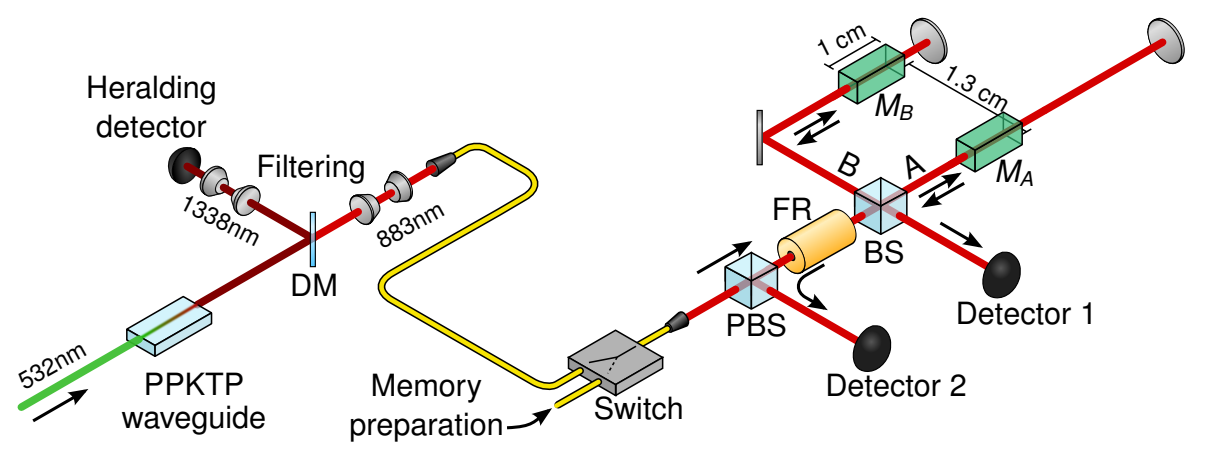

FIG. 1. Experimental setup. The quantum memories $M_{A}$ and $M_{B}$ are implemented using neodymium ions doped into yttrium ortho-silicate crystals $\left(\mathrm{Nd}^{3+}: \mathrm{Y}_{2} \mathrm{SiO}_{5}\right)$ separated by $1.3 \mathrm{~cm}$ and cooled to $3 \mathrm{~K}$ using a cryostat (see ref. [24] for details). The total efficiency of each memory was $15 \%$. A fiber optic switch is used to alternate between a $15 \mathrm{~ms}$ long preparation of the two $\mathrm{Nd}$ ensembles as atomic frequency combs on the ${ }^{4} \mathrm{~F}_{3 / 2} \rightarrow{ }^{4} \mathrm{I}_{9 / 2}$ transition, followed by attempts of entanglement creation for another $15 \mathrm{~ms}$. For the latter, continuous wave light at $532 \mathrm{~nm}$ is coupled into a periodically poled KTP waveguide, leading to the production of pairs of photons at wavelengths of $883 \mathrm{~nm}$ and $1338 \mathrm{~nm}$ through spontaneous parametric downconversion. Photons from each pair are separated on a dichroic mirror (DM) and frequency filtered to below the $120 \mathrm{MHz}$ bandwidth of the quantum memories. The detection of an idler photon at $1338 \mathrm{~nm}$ (using a low-noise superconducting single photon detector [27]) heralds the presence of a signal photon at $883 \mathrm{~nm}$. The signal photon now traverses the switch, a polarizing beam splitter (PBS) and a Faraday rotator (FR), before a 50/50 beamsplitter (BS) creates single-photon entanglement between spatial modes $A$ and $B$. This entanglement is, upon absorption, mapped onto the crystals $M_{A}$ and $M_{B}$. After a preprogrammed storage time of $33 \mathrm{~ns}$, the photons are reemitted and pass through the BS again. Depending on which output mode of BS they emerge from, they either reach detector 1 or are rotated in polarization by the FR and reflected by the PBS towards detector 2 .

optical depth and the quantum memory efficiency up to $15 \%$. The resulting fields can then be probed using single photon detectors to reveal heralded entanglement between the memories. Since the entanglement cannot increase through local operations on the optical modes $A$ and $B$, the entanglement of the retrieved light fields provides a lower bound for the entanglement between the two memories.

The photonic state retrieved from the memories is described by a density matrix $\rho$, including loss and noise, expressed in the Fock state basis. To reveal entanglement in this basis, one cannot resort to violating a Bell inequality given solely inefficient and noisy single photon detectors. Instead, as shown in ref. [5] (see also ref. [13, 14]), a tomographic approach based on single photon detectors can be used. Indeed, it is possible to determine the presence of entanglement between the retrieved fields from the knowledge of the heralded probabilities $p_{m n}$ of detecting $m$ photons in mode $A$ and $n$ in mode $B$, where $m, n \in\{0,1\}$, and from the coherence between these modes. More precisely, one can obtain a lower bound on the concurrence $C$ of the detected fields, a measure of entanglement ranging from 0 for a separable state to 1 for a maximally entangled state, through

$$
C \geq \max \left(0, V\left(p_{01}+p_{10}\right)-2 \sqrt{p_{00} p_{11}}\right) .
$$

The term $V$ is the interference visibility obtained by recombining optical modes $A$ and $B$ on a $50 / 50$ beamsplitter: it is directly proportional to the coherence between the retrieved fields in modes $A$ and $B$. To obtain a large concurrence, that is, a large amount of entanglement, one should maximize $V$ (the coherence) and $p_{10}+p_{01}$ (the probability to detect the heralded photon), and minimize $p_{00}$ and $p_{11}$ (the probabilities of detecting separable states $|0\rangle_{A}|0\rangle_{B}$ and $|1\rangle_{A}|1\rangle_{B}$ stemming from a lost signal photon and from two signal photons, respectively). Implicit to this method is the assumptions that i) the creation of more than two pairs is negligible, and ii) the off-diagonal elements of $\rho$ with different number of photons vanish (this is valid since no local oscillator providing a phase reference was used; see ref. [5]).

To estimate $V, p_{00}, p_{10}, p_{01}$ and $p_{11}$, we used the setup of Fig. 1 in the following way. First, the visibility $V$ was measured by allowing the re-emitted delocalized photon to interfere with itself using a balanced Michelson interferometer whose phase was actively stabilized (see Fig. 1). Then, we blocked spatial mode $B$ to estimate $p_{10}$ by summing the number of detections on detectors 1 and 2 , conditioned on a heralding signal. Probability $p_{01}$ is estimated similarly by blocking mode $A$. Note that the probability of getting simultaneous counts on both detectors was negligibly small in front of the probability of a single detection. Then, $p_{00}$ is estimated through normalization of the total probability, $p_{00}+p_{10}+p_{01} \approx 1$, which is justified by the fact that $p_{11} \ll p_{10}+p_{01} \ll p_{00}$ (see measured values in the Appendix). To estimate $p_{11}$, we used two different methods, described below.

In the first method, we use a direct measurement of threefold coincidences, i.e. involving all three detectors. Details about this measurement are given in the Appendix. With a pump power of $16 \mathrm{~mW}$ and a coincidence time window of $10 \mathrm{~ns}$, we obtained $C^{(\mathrm{MLE})}=$ $6.3(3.8) \times 10^{-5}$ using a maximum likelihood estimation (MLE) of the threefold coincidence probability, and 
$C^{(\mathrm{CE})}=3.9(3.8) \times 10^{-5}$ using a more conservative estimation (CE); see Appendix. Both estimations yield a concurrence that is greater than 0 by at least one standard deviation, and show that entanglement was indeed present between the two crystals. This measurement required 166 hours, a period in which two threefold coincidences were observed. The prohibitively long integration time of this method prevented us from attempting it with lower pump powers (i.e. for lower probability of creating more than one pair). Hence, to study how the concurrence changes with pump power, we used a second method based on twofold coincidences, which we now describe.

In the second method, $p_{11}$ is estimated using a supplementary assumption (see the Appendix for details). This approach is motivated by the results obtained in ref. 13]. Specifically, we assume that all the observed detections stem from a two-mode squeezed-state, and thus the measured zero-time cross-correlation $\bar{g}_{s, i}$ can be written as $\bar{g}_{s, i}=1+1 / p$, where $p \ll 1$ and $p^{2}$ are interpreted as the probabilities of creating one and two photon pairs, respectively. We then proceed as follows to estimate $p_{11}$. We first measure the zero-time crosscorrelation $g_{s, i}^{A}$ between detections in the idler mode and the signal mode $A$ (with mode $B$ blocked). Then we measure $g_{s, i}^{B}$ in the same way (with mode $A$ blocked) and verify that $g_{s, i}^{A} \approx g_{s, i}^{B}$. We calculate the average of $g_{s, i}^{A}$ and $g_{s, i}^{B}$, denoted $\bar{g}_{s, i}$, and estimate $p_{11}$ using

$$
p_{11}=\frac{4 p_{10} p_{01}}{\bar{g}_{s, i}-1} .
$$

In the Appendix, we provide justifications and additional measurements that support our assumption and give evidence that it yields a lower bound on the concurrence. In particular, we measured the second-order autocorrelation of the signal (or idler) mode without storage to be $g_{s, s}^{(2)}(0)=1.81(2)\left(\right.$ or $\left.g_{i, i}^{(2)}(0)=1.86(9)\right)$, which is very close to the ideal value of 2 associated with the thermal photon statistics of a two-mode squeezed state. We also measured the zero-time second-order autocorrelation function of the heralded signal photon just before storage and obtained $g_{s, s \mid i}^{(2)}(0)=0.061$ (4) for a pump power of $8 \mathrm{~mW}$, which is consistent with $p \ll 1$. Note that this method of estimating $p_{11}$ is based on twofold coincidences as opposed to threefold coincidences for the other method. For our specific setup, this resulted in a reduction of the measurement time by a factor of $10^{6}$ for similar statistical confidence on the concurrence. This method requires no physical modifications to the optical circuit to measure the different components of the retrieved fields, which simplifies its implementation.

We performed a series of measurements for several values of the pump power, which is proportional to $p$ provided $p \ll 1$. For all measurements we used coincidence windows of $10 \mathrm{~ns}$, and all results are based on raw counts (i.e. without subtraction of dark counts and accidental coincidences). The inset of $2 \mathrm{a}$ shows the interference of a

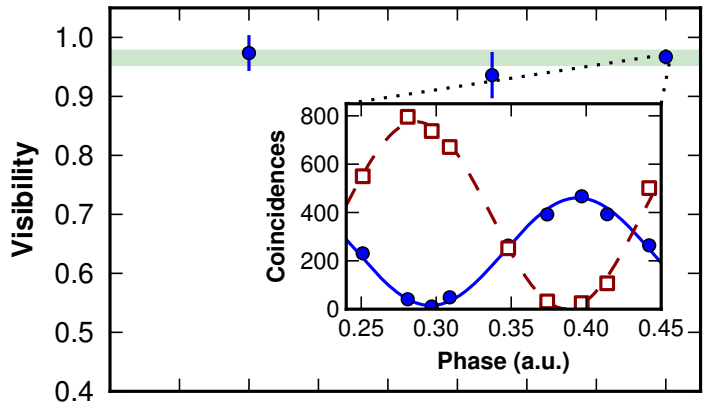

b

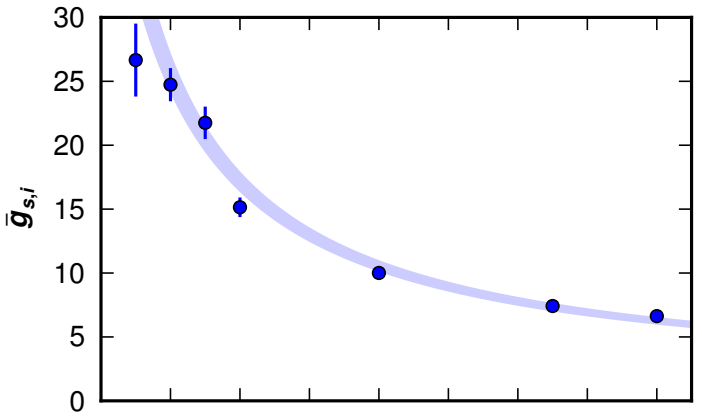

C

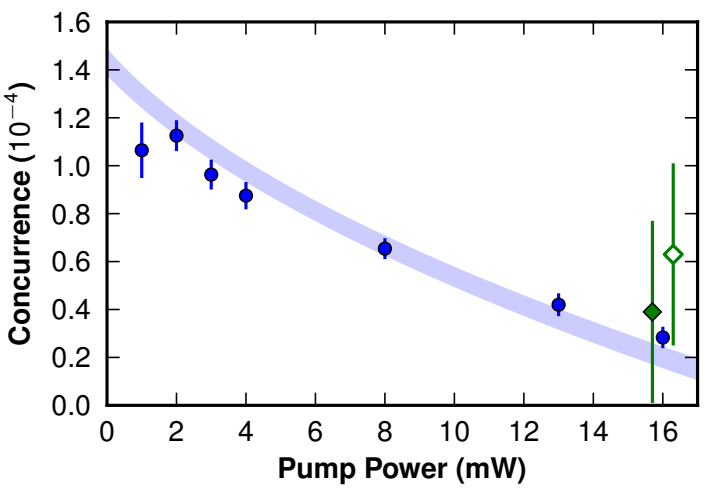

FIG. 2. Results. a, Visibility as a function of pump power. The visibility is approximately constant with an average of $96.5 \pm 1.2 \%$ (green shaded region). The inset shows the visibility curves at $16 \mathrm{~mW}$ measured with detectors $1(\bullet)$ and 2 (ㅁ) (15 minutes acquisition time per point; the error bars are smaller than the symbols). The different amplitudes result from non-uniform loss after recombination of modes $A$ and $B$ on the beamsplitter. The visibilities agree within uncertainties in the fits. $\mathbf{b}$, Zero-time cross-correlation $\bar{g}_{s, i}$ as a function of pump power. The decreasing values agree well with a theoretical model (shaded region; see Appendix). c, Lower bound on the concurrence estimated using the cross-correlation measurement the as a function of pump power $(\mathbf{\bullet})$, calculated using Eq.1 and 2 and the mean visibility of $\mathbf{a}$. The concurrence decreases with pump power, as expected, but remains positive up to $16 \mathrm{~mW}$. The shaded region corresponds to the model in b. All measured values of $p_{10}, p_{01}$ and $p_{11}$ are given in the Appendix. All values are based on raw counts. Uncertainties are obtained assuming Poissonnian detection statistics. The lower bound on the concurrence at $16 \mathrm{~mW}$ obtained from measured threefold coincidences using either the maximum likelihood estimation $(\diamond)$ or the conservative estimation $(\diamond)$ are also shown (they are horizontally offsetted for clarity). 
the delocalized single photon retrieved from the memories as a function of the phase of the interferometer. The visibilities obtained with detectors 1 and 2 agree within the uncertainty of the fit. As shown on Fig. 2a, the visibility does not depend on the pump power and has an average of 96.5(1.2)\%, implying almost perfect coherence between the two memories and a large signal-to-noise ratio. Fig. $2 \mathrm{~b}$ shows the measured $\bar{g}_{s, i}$. Reducing the pump power increases the cross-correlation, as expected. Using additional measurements, we estimated the transmission loss, memory efficiency, dark count probability and the pair creation probability of our setup. These values were then used in a theoretical model (shaded region on Fig. 2b) that is in excellent agreement with the measured values of $\bar{g}_{s, i}$. As explained in the Appendix, this agreement provides evidence that the estimated value of $p_{11}$ yields a lower bound on the concurrence. Finally, Fig. 2c shows the lower bound on the concurrence for all pump powers, calculated using Eqs. 11 and 2 and the average visibility of Fig. 2a. The concurrence decreases with pump power because $p_{11}$ increases, while all other terms of Eq. 1 depend on photon loss only and hence are approximately constant. Nevertheless, the concurrence stays positive for all used pump powers, proving the creation of heralded entanglement between the atomic ensembles inside the two crystals. The theoretical curve on Fig. 2c (shaded region) is based on the same model used for the theoretical curve in Fig. $2 b$.

The results of the measurement of the concurrence based on threefold coincidences are plotted on Fig. 2c and agree, within uncertainty, with the result of the method based on measurement of the cross-correlation.

The observed values of the concurrence are lower bounds on the amount of entanglement of the detected fields and are almost entirely determined by optical loss. To see this, we first note that when both the multi-pair creation probability and the total transmission probability $\eta$ of the signal photon are small, the concurrence is approximately given by $C \approx \eta\left(V-2 / \sqrt{\bar{g}_{s, i}-1}\right)$. At $8 \mathrm{~mW}$ of pump power, we measured $g_{s, i} \approx 10, p_{00}=$ $0.9997831(71)$, and $p_{11}=5.18(40) \times 10^{-9}$, and hence $C \approx 0.3 \eta$. With $\eta \approx p_{10}+p_{01}=2.2 \times 10^{-4}$, we see that $C \approx 6.6 \times 10^{-4} \ll 0.3$. Using this, an estimate of the concurrence of the fields retrieved just after the crystals is obtained by noticing that the transmission $\eta$ is the product of the probability to find the signal photon inside the fiber per heralding signal (20\%), the memory efficiency $(15 \%)$, the transmission in the interferometer $(2.4 \%)$ and detector efficiency $(30 \%)$. Subtracting detector inefficiency and interferometer loss yields a lower bound of approximately 0.092 for the concurrence of the field retrieved just after crystals.

The high interference visibility indicates that the stored entanglement does not significantly decohere during the $33 \mathrm{~ns}$, and the coherent nature of the storage endures for longer times, as shown previously using storage of weak coherent states [22, 30. Increasing the storage time does however lower the cross-correlation func- tion $\bar{g}_{s, i}$, as measured in ref. [24], and this should consequently lower the concurrence of the stored entanglement (this is essentially due to the decrease of the memory efficiency with increasing storage times, as shown in the Appendix and in ref. 24]). With $\mathrm{Nd}^{3+}: \mathrm{Y}_{2} \mathrm{SiO}_{5}$, one could in principle store entanglement up to $\sim 1 \mu$ s (see ref. [22]). A promising approach to go beyond these limits, and to allow on-demand retrieval of the stored entanglement, is to implement spin-wave storage 29] to increase storage times (as demonstrated recently 31 in $\mathrm{Pr}^{3+}: \mathrm{Y}_{2} \mathrm{SiO}_{5}$ ), and to place the crystal inside an impedance-matched cavity to increase the efficiency 34]. Such improvements are necessary for the development of a quantum repeater based on solid-state quantum memories, and are the subject of current research.

In conclusion, we have reported an experimental observation of heralded quantum entanglement between two separate solid-state quantum memories. We emphasize that although the entangled state involves only one excitation, the observed entanglement shows that the stored excitation is coherently delocalized among all the neodymium ions in resonance with the photon, meaning roughly $10^{10}$ ions in each crystal. Our results demonstrate that rare-earth-ion ensembles, naturally trapped in crystals, have the potential of compact, stable and coherent quantum network nodes. Moreover, single photon entanglement is a simple form of entanglement which can be used for teleportation 35] and entanglement swapping operations 3. and can also be purified using linear optical elements [36. It is also a critical resource in several proposals for quantum repeaters [3, 26, 37] since it is less sensitive to transmission loss and detector inefficiencies 44 as compared to two-photon entangled states.

Our experimental approach is based on solid-state devices, the key components being the PPKTP chip with an integrated waveguide (the photon source) and the crystal memory. We believe this approach opens possibilities of integration of components, for instance frequency filtering directly on the chip [38, or waveguide quantum memories 25]. The prospect of combining solid-state photon sources and quantum memories is attractive for practical future quantum networks. One important challenge in this context is to create entanglement between two remote solids in a heralded way, which would realize an elementary link for quantum repeaters.

\section{ACKNOWLEDGMENTS}

We thank H. de Riedmatten, P. Sekatski, and J. Laurat for stimulating discussions, and A. Korneev for help with the superconducting detector. This work was supported by the Swiss NCCR Quantum Science Technology, the Science and Technology Cooperation Program Switzerland-Russia, as well as by the European projects QuRep and ERC-Qore. F.B. was supported in part by le Fond Québécois de la Recherche sur la Nature et les Technologies. 


\section{APPENDIX}

This appendix provides details on the method we used to estimate the threefold coincidence probability $p_{11}$ using either the cross-correlation $g_{s, i}$ or by direct measurement. Specifically, in section A, we present a model describing our setup composed of the source of photon pairs and the memories, and includes the effect of dark counts. We also detail additional measurements that support our model. We present evidence that the method based on the measurement of $g_{s, i}$ to estimate $p_{11}$ yields a lower bound on the concurrence at detection and, consequently, on the concurrence of the delocalized excitation stored in the quantum memories. In section $B$, we provide details on our method to estimate $p_{11}$ directly, we show how the uncertainty on the threefold coincidence probability is calculated and we show the results.

\section{Appendix A: Concurrence using the cross-correlation}

Let us begin by a description of the source. In our model, the SPDC source produces a two-mode squeezed state (where the coherences are lost due to the lack of a phase reference)

$$
\cosh ^{-2} r \sum_{n=0}^{+\infty} \tanh ^{2 n} r\left|n_{i} n_{s}\right\rangle\left\langle n_{i} n_{s}\right|
$$

Here, $n_{i}$ and $n_{s}$ correspond to the number of photons in the idler and signal modes (and are both equal to $n$ ). While the signal and idler fields are perfectly correlated in photon number, they individually exhibit thermal statistics and hence, their second-order auto-correlation function $g^{(2)}(0)$ is, in theory, equal to 2. We experimentally tested this for the photons emitted by the source by performing direct measurements of $g_{i, i}^{(2)}(\tau), g_{s, s}^{(2)}(\tau)$ and $g_{s, s \mid i}^{(2)}(\tau)$, see Fig. A3. The latter is the auto-correlation function of the signal conditioned on the detection of an idler photon at time $\tau$. For the measurements we added $50 / 50$ beam splitters after the frequency filters of the signal or idler photon, respectively, and registered coincidence events using free running detectors. We found zero-delay auto-correlations of $g_{i, i}^{(2)}(0)=1.86(9)$ and $g_{s, s}^{(2)}(0)=1.81(2)$, which are very close to the expected values. This is consistent with the assumption that our source produces a state of the form of Eq. (A1). Additionally, we found a conditional auto-correlation of the signal photon at zero time delay of $g_{s, s \mid i}^{(2)}(0)=0.061(4)$ at a pump power of $8 \mathrm{~mW}$. This shows the very good single photon character of the heralded signal fields, and that $\tanh ^{2} r \ll 1$.

Let us continue the description of our model by focusing on the memory. Due to non-unit absorption efficiency, each signal photon detection after the memory a

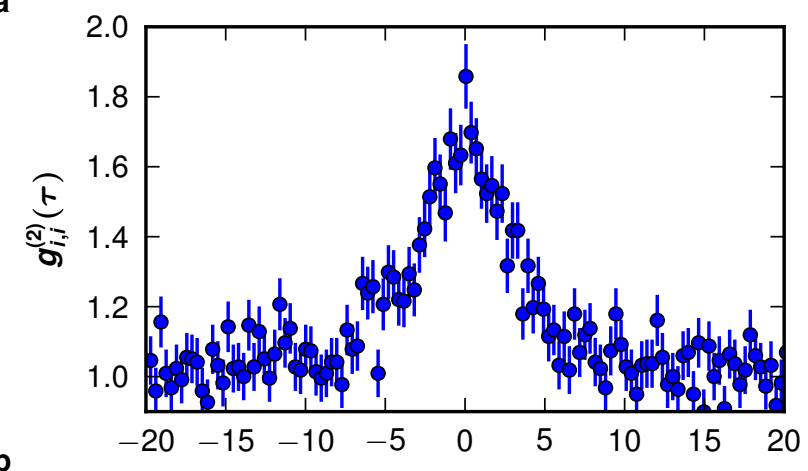

b

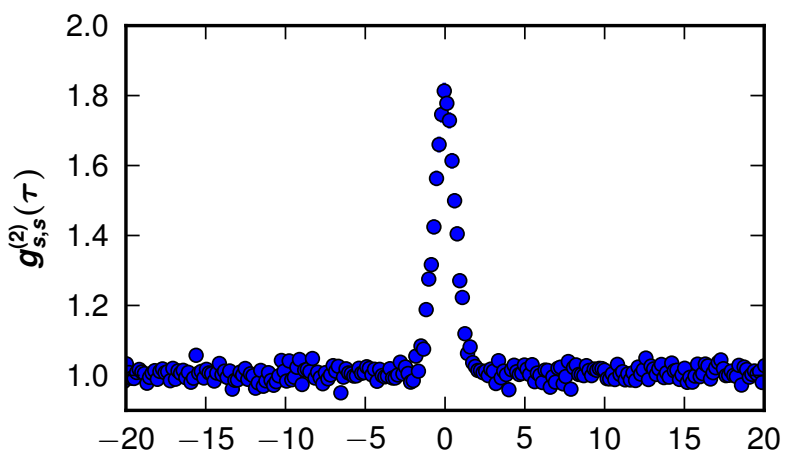

C

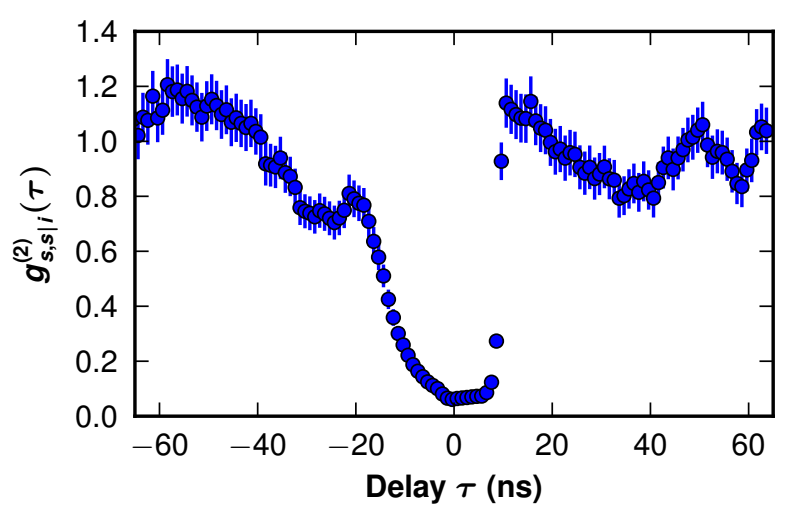

FIG. A3. Measurements of second-order auto-correlation bunctions of $\mathbf{a}$, the idler photon $(1338 \mathrm{~nm}), \mathbf{b}$, the signal photon $(883 \mathrm{~nm})$ before it is sent towards the crystals, and $\mathbf{c}$, the same as $\mathbf{b}$ but conditioned on a coincidence detection of an idler and the first signal photon at delay $\tau=0$. The values at zero delay in $\mathbf{a}$ and $\mathbf{b}$ are close to the theoretical value of 2 . The almost zero minimum value in $\mathbf{c}$ gives evidence of the single photon character of the heralded signal photon. Data was accumulated for 72 hours at a pump power of $16 \mathrm{~mW}$ in $\mathbf{a}$, and for 12 hours at $8 \mathrm{~mW}$ in $\mathbf{b}$ and $\mathbf{c}$. The values in $\mathbf{c}$ have been calculated for a coincidence window of 10 ns. Error bars are due to Poisson counting statistics.

stems either from a photon created at an early time, stored in the medium, and later retrieved; or from a photon created at a later time and not absorbed by the medium. Hence, we need to consider two time bins. The storage process is a linear operation, mapping the light field onto an atomic state. Thus, in our model, it can be represented by a beamsplitter with a transmission cor- 


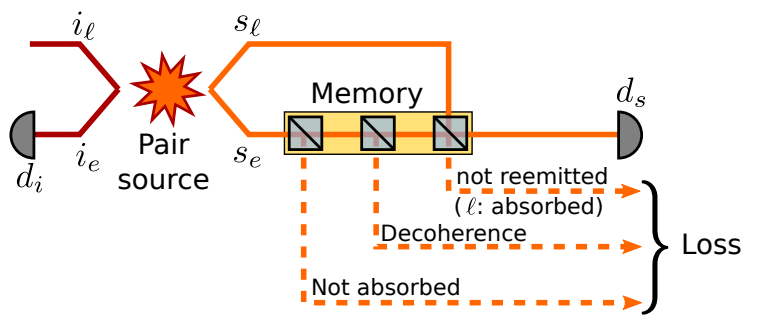

FIG. A4. Model of our experimental setup. The source produces idler-signal pairs described by a two-mode squeezed state. The memory is represented by three beamsplitters (to account for the absorption, the decoherence and the reemission respectively). Each temporal mode after the memory $d_{s}$ comes either from a signal photon emitted at an early time, then stored and later retrieved (mode $s_{e}$ ), or from a photon produced at a late time and directly transmitted through the memory (mode $s_{\ell}$ ). To take both contributions into account, a virtual source creating idler-signal pairs in a late time bin has been introduced. The correlations between the modes $d_{s}$ and $d_{i}$ are characterized through the measurement of the second-order cross-correlation function.

responding to the absorption probability, c.f. Fig. A4. Another beamsplitter is introduced to account for the decoherence, inherent to the storage process 29. Finally, a last beamsplitter accounts for the reemission of the photon. It is identical to the first one and combines the two time bins into a single temporal mode $d_{s}$. The output of the last beamsplitter is detected to access the cross-correlation function

$$
\bar{g}_{s, i}=\frac{\left\langle d_{i}^{\dagger} d_{i} d_{s}^{\dagger} d_{s}\right\rangle}{\left\langle d_{i}^{\dagger} d_{i}\right\rangle\left\langle d_{s}^{\dagger} d_{s}\right\rangle}
$$

between detection on the early idler mode $d_{i}$ and signal mode $d_{s}$. Note that the overall transmission (in intensity) of the three beamsplitters corresponds to the efficiency of the storage and retrieval processes $\eta_{\text {echo }}$, while the reflection of the last beamsplitter is the transmission of the atomic ensemble $\eta_{\text {trans }}$.

Using the Heisenberg picture

$$
\left\langle d_{s}^{\dagger} d_{s}\right\rangle=\left\langle 0\left|U^{\dagger} d_{s}^{\dagger} U U^{\dagger} d_{s} U\right| 0\right\rangle
$$

where

$$
\begin{aligned}
U^{\dagger} d_{s} U & =\sqrt{\eta_{\text {echo }}}\left(\cosh r s_{e}+\sinh r i_{e}^{\dagger}\right) \\
& +\sqrt{\eta_{\text {trans }}}\left(\cosh r s_{\ell}+\sinh r i_{\ell}^{\dagger}\right)
\end{aligned}
$$

leading to

$$
\left\langle d_{s}^{\dagger} d_{s}\right\rangle=\left(\eta_{\text {trans }}+\eta_{\text {echo }}\right) \sinh ^{2} r .
$$

Furthermore, we have

$$
U^{\dagger} d_{i} U=\left(\cosh r i_{e}+\sinh r s_{e}^{\dagger}\right)
$$

leading to

$$
\left\langle d_{i}^{\dagger} d_{i}\right\rangle=\sinh ^{2} r
$$

Following similar lines, we find

$$
\begin{aligned}
\left\langle d_{i}^{\dagger} d_{i} d_{s}^{\dagger} d_{s}\right\rangle=\sinh ^{2} r & \left(\sinh ^{2} r\left(\eta_{\text {trans }}+\eta_{\text {echo }}\right)\right. \\
& \left.+\cosh ^{2} r \eta_{\text {echo }}\right)
\end{aligned}
$$

Therefore, the cross-correlation function between the idler-signal modes is given by

$$
1+\frac{1}{\tanh ^{2} r\left(1+\frac{\eta_{\text {trans }}}{\eta_{\text {echo }}}\right)} .
$$

Note that the effect of the memories is to add the term $\left(1+\frac{\eta_{\text {trans }}}{\eta_{\text {echo }}}\right)$, which decreases the cross-correlation. One can further take the detector noise into account by adding an additional source of noise with a Poissonian photon distribution. We finally find

$$
g_{s, i}=1+\frac{1}{\tanh ^{2} r\left(1+\frac{\eta_{\text {trans }}}{\eta_{\text {echo }}}\right)+\frac{\eta_{\text {dark }}}{p_{c}}}
$$

where $\eta_{\text {dark }}$ is the probability to get a dark count within the detection window and $p_{c}$ is the conditional probability of detecting the signal photon retrieved from the memory. Note that for small pump powers, the probability to create one pair is given by

$$
\tanh ^{2} r / \cosh ^{2} r \approx r^{2}=\alpha P_{\text {pump }},
$$

where $P_{\text {pump }}$ is the continuous wave pump power.

To support the model presented above, we performed additional measurements to determine the values of $\alpha$, $\eta_{\text {trans }} / \eta_{\text {echo }}, p_{c}$ and (see Table A1). First, we estimated $\alpha=2.71(8) \times 10^{-3}$ pairs $/ \mathrm{mW}$ from a measurement of the cross-correlation as function of pump power with one crystal prepared as a transmission window (no storage) while the path of other crystal was blocked. Then, $\eta_{\text {trans }} / \eta_{\text {echo }}$ was measured from the ratio of the probabilities of detecting the heralded signal photon when it is either transmitted through the memory (no storage), or stored and retrieved from the memory. This measurement was performed twice - once for each arm of the interferometer (with the other arm blocked) - and the outcomes were averaged. The conditional probability $p_{c}$ was taken as the average of probabilities $p_{10}$ and $p_{01}$ of detecting the heralded signal photon retrieved from the memories (see main text). Finally, we measured a dark count probability of $\eta_{\text {dark }}=2 \times 10^{-6}$ per 10 ns detection window for the signal mode detector. Idler photons were detected using a superconducting nanowire single photon detector with a dark count rate of $20 \mathrm{~Hz}$. This low dark count rate is neglected in our calculations. Using these parameters, we compared the measured crosscorrelation $\bar{g}_{s, i}$ with values of $g_{s, i}$ predicted by Eq. A3. The comparison is shown on Fig. $2 \mathrm{~B}$ in the main text. We find excellent agreement within the statistical uncertainties, thereby supporting our model for the source and the memory. 
TABLE A1. Conditional probabilities $p_{01}, p_{10}, p_{11}$ and the ratio $\eta_{\text {trans }} / \eta_{\text {echo }}$ for various pump powers.

\begin{tabular}{lllll}
\hline $\begin{array}{l}\text { Pump } \\
\text { power }\end{array}$ & $p_{01}\left(10^{-4}\right)$ & $p_{10}\left(10^{-4}\right)$ & $p_{11}\left(10^{-9}\right)$ & $\frac{\eta_{\text {trans }}}{\eta_{\text {echo }}}$ \\
\hline $1 \mathrm{~mW}$ & $1.04(14)$ & $0.82(12)$ & $1.33(30)$ & $2.84(33)$ \\
$2 \mathrm{~mW}$ & $1.193(75)$ & $0.809(63)$ & $1.63(19)$ & $3.03(17)$ \\
$3 \mathrm{~mW}$ & $0.952(72)$ & $0.878(70)$ & $1.61(20)$ & $2.59(17)$ \\
$4 \mathrm{~mW}$ & $1.105(72)$ & $0.902(66)$ & $2.82(31)$ & $3.35(19)$ \\
$8 \mathrm{~mW}$ & $1.185(51)$ & $0.984(50)$ & $5.18(40)$ & $3.13(12)$ \\
$13 \mathrm{~mW}$ & $1.247(56)$ & $1.131(52)$ & $8.79(66)$ & $2.86(11)$ \\
$16 \mathrm{~mW}$ & $1.146(47)$ & $1.175(48)$ & $9.56(64)$ & $2.748(93)$ \\
\hline Mean & $1.123(30)$ & $0.957(27)$ & & $2.936(69)$ \\
\hline
\end{tabular}

We now give evidence based on our model that the method we used to estimate the threefold coincidence probability leads to an underestimation of the concurrence. For this, we compare the threefold coincidence probability $p_{11}^{t h}$ predicted by our model with the one obtained from the method based on the measured crosscorrelation $\bar{g}_{s, i}$. Let us first concentrate on $p_{11}^{t h}$. Using our model, we can predict that the threefold coincidence probability should be given by

$$
p_{11}^{t h}=4 p_{10} p_{01}\left[\alpha P_{\text {pump }}\left(1+\frac{\eta_{\text {trans }}}{2 \eta_{\text {echo }}}\right)+\frac{\eta_{\text {dark }}}{p_{c}} .\right] .
$$

This should be compared with the threefold coincidence probability estimated using $\bar{g}_{s, i}=1+1 / p$, i.e. assuming detections stem from a two-mode squeezed states (see main text). Combining this method with our model (Eq. A3) yields

$$
\begin{aligned}
p_{11} & =\frac{4 p_{10} p_{01}}{\bar{g}_{s, i}-1} \\
& =4 p_{10} p_{01}\left[\alpha P_{\text {pump }}\left(1+\frac{\eta_{\text {trans }}}{\eta_{\text {echo }}}\right)+\frac{\eta_{\text {dark }}}{p_{c}}\right] .
\end{aligned}
$$

One can see that the ratio $\eta_{\text {trans }} / \eta_{\text {echo }}$ enters in $p_{11}$ without the prefactor of one-half, such that $p_{11}>p_{11}^{t h}$. From Eq. (1) of the main text, we directly see that an overestimation of $p_{11}$ leads to a lower bound on the concurrence at detection, and hence on the concurrence of the entanglement stored in the memories.

\section{Appendix B: Concurrence using a direct measurement of the threefold coincidence probability}

\section{Method}

We now describe how we estimate the probability $p_{11}$ from the measurement of threefold coincidences. For this, we use the experimental setup shown on Fig. A5 this is the same setup shown on Fig. 1 of the main text. Unlike our estimation based on the measurement of the crosscorrelation (section $\mathrm{A}$, this direct method does not re- quire assumptions on the statistics of the source of photon pairs and on how the memories operate.

Let us consider the two-photon part, denoted $\rho_{2}$, of the complete density matrix describing the state of the retrieved fields just after the crystals. If the relative phase between the spatial modes is randomized, then the coherences are zero and we can write

$$
\rho_{2}=q_{11}|11\rangle\left\langle 11\left|+q_{20}\right| 20\right\rangle\left\langle 20\left|+q_{02}\right| 02\right\rangle\langle 02| .
$$

Here, $q_{11}$ is the probability per heralding signal that the fields retrieved from crystals $\mathrm{A}$ and $\mathrm{B}$ each contain one photon, $q_{20}\left(q_{02}\right)$ the probability per heralding signal that the fields retrieved from crystals $\mathrm{A}$ and $\mathrm{B}$ each contain 2 and 0 (0 and 2) photons, respectively. Estimation of $p_{11}$ can be performed by measuring the $q_{11}$ probability in Eq. A1, as in Ref. [5]. We show here that one can also estimate $p_{11}$ by measuring $q_{20}$ and $q_{02}$.

We first derive useful relations between the occupation probabilities. We use the following definitions: $P_{H}$ is the probability to herald the presence of a signal photon (i.e. the probability to detect an idler photon during the detection time window); $\eta_{\text {echo }}^{A}\left(\eta_{\text {echo }}^{B}\right)$ is the storage and retrieval efficiency of crystal $\mathrm{A}(\mathrm{B}) ; P_{2}$ is the probability that the source of photon pair emits 2 pairs of photons during the detection time window; $R$ and $T$ are the intensity reflection and transmission coefficients of the beamsplitter when photons are incident from the left hand side, satisfying $R+T \leq 1$ and $R, T>0$ (see Fig. A5. Using these definitions, and the linear nature of the optical storage process, we have

$$
\begin{aligned}
q_{11} & =\frac{1}{P_{H}} 2 R T \eta_{\text {echo }}^{A} \eta_{\text {echo }}^{B} P_{2}, \\
q_{20} & =\frac{1}{P_{H}} T^{2}\left(\eta_{\text {echo }}^{A}\right)^{2} P_{2}, \\
q_{20} & =\frac{1}{P_{H}} R^{2}\left(\eta_{\text {echo }}^{B}\right)^{2} P_{2},
\end{aligned}
$$

with which we can write $q_{20}$ and $q_{02}$ as a function of $q_{11}$ :

$$
\begin{aligned}
& q_{20}=q_{11}(R / 2 T), \\
& q_{02}=q_{11}(T / 2 R) .
\end{aligned}
$$

We use these expressions later.

We now consider recombination at the beamsplitter. We describe the effect of the beamsplitter on mode operators $a$ and $b$ as follows:

$$
\begin{aligned}
& a^{\dagger} \rightarrow \alpha_{t} a^{\dagger}+\alpha_{r} b^{\dagger}+\alpha_{l} l^{\dagger}, \\
& b^{\dagger} \rightarrow \beta_{r} a^{\dagger}+\beta_{t} b^{\dagger}+\beta_{l} l^{\dagger},
\end{aligned}
$$

where mode $l$ represents loss. We set all coefficients to be real. Energy conservation yields $\alpha_{r}^{2}+\alpha_{t}^{2}+\alpha_{l}^{2}=1$ (and similarly for the coefficients of mode $b$ ). Also, unitarity imposes that $\alpha_{r} \alpha_{t}+\beta_{r} \beta_{t}=0$. Finally, we suppose that $0<\alpha_{t}^{2}, \alpha_{r}^{2}, \beta_{t}^{2}, \beta_{r}^{2} \leq 1 / 2$, and we note that $R=\beta_{r}^{2}$ and $T=\alpha_{t}^{2}$. Using these notations, we can show that the probability to get a coincidence at detectors 1 and 2 per heralding signal is given by

$$
\bar{p}_{11}=\left(a_{11} q_{11}+a_{20} q_{20}+a_{02} q_{02}\right) \eta_{1} \eta_{2},
$$




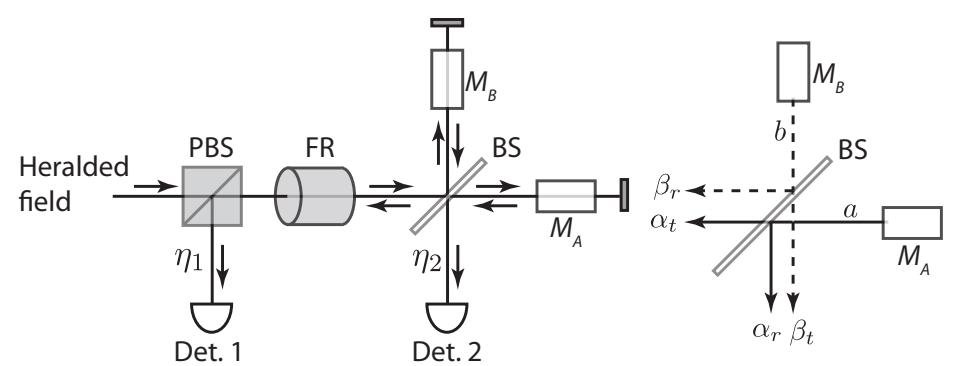

FIG. A5. Experimental setup used to estimate $\bar{p}_{11}$ directly. This setup is the same of Fig. 1 of the main text. The relative phase between the arms of the interferometer was randomized by letting the interferometer (see Fig. A5 drift by itself over the total measurement period of more than 100 hours. PBS: polarizing beamsplitter; FR: Faraday rotator; BS: beamsplitter; $M_{A}$ and $M_{B}$ are crystal memories $\mathrm{A}$ and $\mathrm{B}$. The detection efficiency from the output modes of the beamsplitter to detectors 1 and 2 and $\eta_{1}$ and $\eta_{2}$, respectively. The amplitude transmission and reflection coefficients of modes $a$ and $b$ right after the crystals, through the beamsplitter, are shown.

where $\eta_{1}\left(\eta_{2}\right)$ is the transmission from output mode $a$ (b) - after the beamsplitter - to detector 1 (detector 2), including the detector efficiency. We also have

$$
\begin{aligned}
& a_{11}=\beta_{r}^{2}\left(\alpha_{r}-\frac{\beta_{t}^{2}}{\alpha_{r}}\right)^{2}, \\
& a_{20}=2 \alpha_{t}^{2} \alpha_{r}^{2}, \\
& a_{02}=2 \beta_{t}^{2} \beta_{r}^{2} .
\end{aligned}
$$

We characterized our beamsplitter and obtained $\alpha_{t}^{2}=$ $T=0.479, \alpha_{r}^{2}=0.422, \beta_{t}^{2}=0.482$ and $\beta_{r}^{2}=R=0.409$. These values yield

$$
\begin{aligned}
& a_{11}=0.0028, \\
& a_{20}=0.394, \\
& a_{02}=0.404 .
\end{aligned}
$$

We immediately notice that $a_{11}$ can be neglected with respect to $a_{20}$ and $a_{02}$. This essentially means that whenever a photon is retrieved from each crystal, they bunch with almost certainty and do not significantly contribute to threefold coincidences. It also requires indistinguishability of the retrieved photons, which is consistent with the large single photon interference visibility observed (96.5\%). Combining Eq. A3 and Eq. A4 and writing $a_{20} \approx a_{02} \approx 0.4$, we get

$$
\bar{p}_{11} \approx 0.4 \eta_{1} \eta_{2}\left(q_{20}+q_{02}\right) .
$$

Finally, using Eq. A2, we get

$$
\begin{aligned}
q_{20}+q_{02} & =\left(\frac{R}{2 T}+\frac{T}{2 R}\right) q_{11} \\
& =1.012 q_{11} \\
& \approx q_{11}
\end{aligned}
$$

and thus

$$
\bar{p}_{11} \approx 0.4 \eta_{1} \eta_{2} q_{11} .
$$

We now have to determine how to use Eq. A6 in the calculation of the concurrence. This method should give a result consistent with the way we measured $p_{10}+p_{01}$, which was done as follows. We let the phase of the interferometer drift to randomize it, and measured the probability to get a detection at detector 1 or 2 per heralding signal. This is equivalent to, first, measure crystal A (with the path of crystal B blocked) using an effective detector of efficiency $\eta_{A}=\alpha_{t}^{2} \eta_{1}+\alpha_{r}^{2} \eta_{2}$, and then measure crystal B (with the path of crystal A blocked) using an effective detector of efficiency $\eta_{B}=\beta_{r}^{2} \eta_{1}+\beta_{t}^{2} \eta_{2}$, and then add the results together. Hence, if both crystals were measured simultaneously and separately, the threefold coincidence probability per heralding signal would be

$$
q_{11} \eta_{A} \eta_{B}
$$

We can now find the relation between Eq. A6 and A7. For this, we use the fact that, in our experiment, $\eta_{2}$ was larger than $\eta_{1}$, which is apparent from the visibility plot in the main text (inset of Fig. 2-A). Hence, by setting $\eta_{1}=\eta_{2} / 2$, we get $q_{11} \eta_{A} \eta_{B} \approx 0.454 \eta_{2}^{2} q_{11}$. Therefore, we immediately see that if we multiply Eq. A6 by 2.27 , we get Eq. A7.

In summary, to estimate the probability $p_{11}$ used in the formula of the concurrence, namely

$$
C \geq \max \left(0, V\left(p_{10}+p_{01}\right)-2 \sqrt{p_{00} p_{11}}\right),
$$

we multiply the measured $\bar{p}_{11}$ by 2.27 . This multiplicative factor was obtained using approximations, and hence has some uncertainty associated to it. Nevertheless, as explained in the next subsection, this uncertainty can be neglected in front of the large statistical uncertainty of $\bar{p}_{11}$.

\section{Statistical uncertainty}

Here we provide details on how the statistical uncertainty on $\bar{p}_{11}$ was calculated in the method based on the measurement of threefold coincidences (subsection B 1). 
We denote $N_{H}$ the number of heralding signals during the measurement period $T, \bar{P}_{11}$ the stochastic variable representing the probability to register a threefold coincidence per heralding signal, and $\bar{p}_{11}$ our estimation of $\bar{P}_{11}$ obtained from the measurement.

Since $\bar{p}_{11} \ll 1$ and $N_{H} \gg 1$, the distribution of the number of coincidences between detectors 1 and 2 per heralding signal (i.e. threefold coincidences) is accurately described by a Poisson distribution. Specifically, the probability to get $n$ coincidences given $N_{H}$ heralding signals is given by

$$
P\left(n \mid \bar{p}_{11}\right)=e^{-N_{H} \bar{p}_{11}} \frac{\left(N_{H} \bar{p}_{11}\right)^{n}}{n !} .
$$

The probability $P\left(n \mid \bar{p}_{11}\right)$ is the likelihood function of $\bar{p}_{11}$ given $n$. Hence, $\bar{P}_{11}$ can be estimated by maximizing this likelihood, which yields

$$
\bar{p}_{11}^{(\mathrm{MLE})}=\frac{n}{N_{H}} \pm \frac{\sqrt{n}}{N_{H}},
$$

where MLE stands for "maximum likelihood estimation". This method yields an appropriate estimate of $\bar{P}_{11}$, unless one measures $n=0$, in which case one would conclude that $\bar{p}_{11}=0 \pm 0$.

A more conservative estimate can be obtained in the following way. Using Bayes' law, we can write the probability density function $\Omega\left(\bar{p}_{11} \mid n\right)$ of getting $\bar{P}_{11}=\bar{p}_{11}$ given $n$ measured threefold coincidences:

$$
\Omega\left(\bar{p}_{11} \mid n\right)=\frac{P\left(n \mid \bar{p}_{11}\right)}{\int_{0}^{1} P\left(n \mid \bar{p}_{11}\right) d \bar{p}_{11}} .
$$

The denominator of Eq. A9 can be calculated:

$$
\begin{aligned}
\int_{0}^{1} e^{-N_{H} \bar{p}_{11}} \frac{\left(N_{H} \bar{p}_{11}\right)^{n}}{n !} d \bar{p}_{11} & =\frac{1}{N_{H}}\left(1-e^{-N_{H}} \sum_{k=0}^{n} \frac{N_{H}^{k}}{k !}\right) \\
& \approx \frac{1}{N_{H}} .
\end{aligned}
$$

The last equality holds because $n \ll N_{H}$. Therefore:

$$
\Omega\left(\bar{p}_{11} \mid n\right)=N_{H} P\left(n \mid \bar{p}_{11}\right) .
$$

Using Eq. A10, we can calculate the expected value of $\bar{P}_{11}$ and its second moment, respectively denoted $\left\langle\bar{P}_{11}\right\rangle$ and $\left\langle\bar{P}_{11}^{2}\right\rangle$ :

$$
\begin{aligned}
& \left\langle\bar{P}_{11}\right\rangle=\int_{0}^{1} \bar{p}_{11} \Omega\left(\bar{p}_{11} \mid n\right) d \bar{p}_{11} \approx \frac{n+1}{N_{H}}, \\
& \left\langle\bar{P}_{11}^{2}\right\rangle=\int_{0}^{1} \bar{p}_{11}^{2} \Omega\left(\bar{p}_{11} \mid n\right) d \bar{p}_{11} \approx \frac{(n+1)(n+2)}{N_{H}^{2}} .
\end{aligned}
$$

The standard deviation of $\bar{P}_{11}$ is therefore

$$
\sqrt{\left\langle\bar{P}_{11}^{2}\right\rangle-\left\langle\bar{P}_{11}\right\rangle^{2}}=\frac{\sqrt{n+1}}{N_{H}} .
$$

Hence, given $n$ measured threefold coincidences and $N_{H}$ heralding signals, this conservative method yields

$$
\bar{p}_{11}^{(\mathrm{CE})}=\frac{n+1}{N_{H}} \pm \frac{\sqrt{n+1}}{N_{H}},
$$

where CE stands for "conservative estimation". In our results, we estimate the concurrence with both the maximum likelihood and the conservative estimations of $\bar{P}_{11}$.

\section{Experimental results}

The estimation of $p_{00}, p_{10}+p_{01}$ and $p_{11}$ proceeded as follows. For a time $T=166$ hours, we let the interferometer drift to randomize the phase. During that period of time, we recorded the total number of heralded twofold coincidences between the heralding detector and detector $1\left(N_{1 \mid H}\right)$, and between the heralding detector and detector $2\left(N_{2 \mid H}\right)$, and calculated $N_{2}=N_{1 \mid H}+N_{2 \mid H}$. We also recorded the total number of heralded threefold coincidences, denoted $N_{12 \mid H}$, between all three detectors. We used a coincidence time window of $10 \mathrm{~ns}$; this is the same value we used in the estimation of the concurrence based on the cross-correlation. We calculated

$$
p_{10}+p_{01}=\frac{N_{2}}{N_{H}} \pm \frac{\sqrt{N_{2}}}{N_{H}} .
$$

For $p_{11}$, we used both the MLE, yielding

$$
p_{11}^{(\mathrm{MLE})}=2.27\left(\frac{N_{12 \mid H}}{N_{H}} \pm \frac{\sqrt{N_{12 \mid H}}}{N_{H}}\right),
$$

and the CE:

$$
p_{11}^{(\mathrm{CE})}=2.27\left(\frac{N_{12 \mid H}+1}{N_{H}} \pm \frac{\sqrt{N_{12 \mid H}+1}}{N_{H}}\right) .
$$

Finally, we estimated $p_{00}=1-p_{10}-p_{01}-p_{11}$.

With a $10 \mathrm{~ns}$ coincidence window, we obtained $p_{10}+$ $p_{01}=1.7777(34) \times 10^{-4}, V=96.5(1.2) \%$, and observed two threefold coincidences $\left(N_{12 \mid H}=2\right)$. This yields

$$
\begin{aligned}
p_{11}^{(\mathrm{MLE})} & =2.9(2.1) \times 10^{-9} \\
C^{(\mathrm{MLE})} & \geq 6.3(3.8) \times 10^{-5}
\end{aligned}
$$

and

$$
\begin{aligned}
p_{11}^{(\mathrm{CE})} & =3.9(2.2) \times 10^{-9} \\
C^{(\mathrm{CE})} & \geq 3.9(3.8) \times 10^{-5}
\end{aligned}
$$

Both the MLE and CE yield a lower bound for the concurrence that is greater than 0 by at least one standard deviation. 
[1] H. J. Kimble, Nature 453, 1023 (2008).

[2] H.-J. Briegel, W. Dür, J. I. Cirac, and P. Zoller, Phys. Rev. Lett. 81, 5932 (1998).

[3] L.-M. Duan, M. D. Lukin, J. I. Cirac, and P. Zoller, Nature 414, 413 (2001).

[4] N. Sangouard, C. Simon, H. de Riedmatten, and N. Gisin, Rev. Mod. Phys. 83, 33 (2011).

[5] C. W. Chou, H. de Riedmatten, D. Felinto, S. V. Polyakov, S. J. van Enk, and H. J. Kimble, Nature 438, 828 (2005).

[6] N. Gisin and R. Thew, Nat Photonics 1, 165 (2007).

[7] V. Giovannetti, S. Lloyd, and L. Maccone, Science 306, 1330 (2004).

[8] M. A. Nielsen and I. L. Chuang, Quantum Computation and Quantum Information (Cambridge, 2000).

[9] P. Kok, W. J. Munro, K. Nemoto, T. C. Ralph, J. P. Dowling, and G. J. Milburn, Rev. Mod. Phys. 79, 135 (2007).

[10] N. Gisin, G. Ribordy, W. Tittel, and H. Zbinden, Rev. Mod. Phys. 74, 145 (2002).

[11] B. Julsgaard, A. Kozhekin, and E. S. Polzik, Nature 413, 400 (2001).

[12] J. Simon, H. Tanji, S. Ghosh, and V. Vuletić, Nat Physics 3, 765 (2007).

[13] J. Laurat, K. S. Choi, H. Deng, C. W. Chou, and H. J. Kimble, Phys. Rev. Lett. 99, 180504 (2007).

[14] K. S. Choi, H. Deng, J. Laurat, and H. J. Kimble, Nature 452, 67 (2008).

[15] D. N. Matsukevich, T. Chaneliere, S. D. Jenkins, S.-Y. Lan, T. A. B. Kennedy, and A. Kuzmich, Phys. Rev. Lett. 96, 030405 (2006).

[16] C. W. Chou, J. Laurat, H. Deng, K. S. Choi, H. de Riedmatten, D. Felinto, and H. J. Kimble, Science 316, 1316 (2007).

[17] Z.-S. Yuan, Y.-A. Chen, B. Zhao, S. Chen, J. Schmiedmayer, and J.-W. Pan, Nature 454, 1098 (2008).

[18] D. L. Moehring, P. Maunz, S. Olmschenk, K. C. Younge, D. N. Matsukevich, L.-M. Duan, and C. Monroe, Nature 449, 68 (2007).

[19] W. Tittel, M. Afzelius, T. Chaneliere, R. Cone, S. Kröll, S. Moiseev, and M. Sellars, Laser \& Photonics Review 4, 244 (2010).

[20] J. J. Longdell, E. Fraval, M. J. Sellars, and N. B. Manson, Phys. Rev. Lett. 95, 063601 (2005).

[21] M. P. Hedges, J. J. Longdell, Y. Li, and M. J. Sellars, Nature 465, 1052 (2010).
[22] I. Usmani, M. Afzelius, H. de Riedmatten, and N. Gisin, Nat Communications 1, 12 (2010).

[23] M. Bonarota, J.-L. L. Gouët, and T. Chanelière, New Journal of Physics 13, 013013 (2011).

[24] C. Clausen, I. Usmani, F. Bussieres, N. Sangouard, M. Afzelius, H. de Riedmatten, and N. Gisin, Nature 469, 508 (2011).

[25] E. Saglamyurek, N. Sinclair, J. Jin, J. A. Slater, D. Oblak, F. Bussieres, M. George, R. Ricken, W. Sohler, and W. Tittel, Nature 469, 512 (2011).

[26] C. Simon, H. de Riedmatten, M. Afzelius, N. Sangouard, H. Zbinden, and N. Gisin, Phys. Rev. Lett. 98, 190503 (2007).

[27] A. Verevkin, A. Pearlman, W. Slysz, J. Zhang, M. Currier, A. Korneev, G. Chulkova, O. Okunev, P. Kouminov, K. Smirnov, B. Voronov, G. N. Gol'tsman, and R. Sobolewski, J. of Mod. Optics 51, 1447 (2004).

[28] S. J. van Enk, Phys. Rev. A 72, 064306 (2005).

[29] M. Afzelius, C. Simon, H. de Riedmatten, and N. Gisin, Phys. Rev. A 79, 052329 (2009).

[30] H. de Riedmatten, M. Afzelius, M. U. Staudt, C. Simon, and N. Gisin, Nature 456, 773 (2008).

[31] M. Afzelius, I. Usmani, A. Amari, B. Lauritzen, A. Walther, C. Simon, N. Sangouard, J. Minar, H. de Riedmatten, N. Gisin, and S. Kröll, Phys. Rev. Lett. 104, 040503 (2010).

[32] M. Sabooni, F. Beaudoin, A. Walther, N. Lin, A. Amari, M. Huang, and S. Kroll, Phys. Rev. Lett. 105, 060501 (2010).

[33] M. Bonarota, J. Ruggiero, J. L. Le Gouët, and T. Chanelière, Phys. Rev. A 81 (2010).

[34] M. Afzelius and C. Simon, Phys. Rev. A 82, 022310 (2010).

[35] D. T. Pegg, L. S. Phillips, and S. M. Barnett, Phys. Rev. Lett. 81, 1604 (1998).

[36] D. Salart, O. Landry, N. Sangouard, N. Gisin, H. Herrmann, B. Sanguinetti, C. Simon, W. Sohler, R. T. Thew, A. Thomas, and H. Zbinden, Phys. Rev. Lett. 104, 180504 (2010).

[37] N. Sangouard, C. Simon, J. Minar, H. Zbinden, H. de Riedmatten, and N. Gisin, Phys. Rev. A 76, 050301 (R) (2007).

[38] E. Pomarico, B. Sanguinetti, N. Gisin, R. Thew, H. Zbinden, G. Schreiber, A. Thomas, and W. Sohler, New Journal of Physics 11, 113042 (2009). 